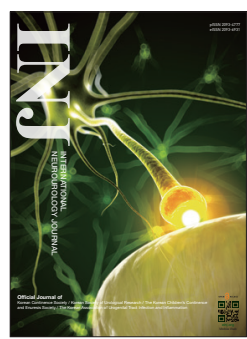

\title{
Learning From Heart Failure: How Will We Lead Bladder Failure Into the Future?
}

\author{
Whi-An Kwon ${ }^{1}$, Tack Lee ${ }^{2}$ (B) http://orcid.org/0000-0001-8508-9099 \\ ${ }^{1}$ Department of Urology, Wonkwang University School of Medicine, Institute of Wonkwang Medical Science, Iksan, Korea \\ ${ }^{2}$ Department of Urology, Inha University Hospital, Incheon, Korea \\ E-mail: lee.tack33@gmail.com
}

Bladder failure is a condition or a collection of symptoms in which the bladder is unable to evacuate the urine completely due to inadequate detrusor contraction [1]. This hidden condition that does not usually lead to complaints is common in the elderly, and its incidence increases inevitably with aging. Moreover, there is a lack of basic research into the underlying causes of this disease, along with a lack of unified terminology and related definitions and acceptable diagnostic criteria. The biggest burden to us, clinicians, is the necessity of requiring incapable elderly patients to perform painful clean intermittent catheterization several times a day.

It is urgent that we restart by coming to a consensus on the terminology of this disease. In cases of heart failure, there seems to be no other currently used terminology for that condition, which might be the first important step towards the current achievements in treating heart failure. This may be the key to the possible differences in the academic content and achievements in the fields of heart failure and bladder failure. The term "bladder failure" still remains surrounded by ambiguity with a lack of accepted terminology [2]. In our opinion, the terminology for any disease should be simple and easily recognizable by the patients as well as physicians. Although "bladder failure" may give the impression of an all-or-nothing event, giving the condition a name may be the first step toward an expansion in the development of bladder failure research.

The heart and bladder are harder-working organs than any others in the body, continuously pumping blood or urine out. The failure of these organs may have similar underlying mecha- nistic causes and pathogenesis. In this issue of International Neurourology Journal, Marquez et al. [3] introduces a close relationship between the heart and mitochondrial function in the regulation of cellular energetics and metabolism, and describes the beneficial effect of targeting mitochondrial biogenesis in failing hearts. Furthermore, although there are currently no pharmacologic agents that directly affect mitochondrial biogenesis in heart failure, this article shows current advances in the development of heart failure therapies, including mitochondrial biogenesis, mitochondrial oxidative stress, and mitochondrial pore transition proteins. A similar relationship between the bladder and mitochondrial function was described previously in a study of bladder failure [4,5]. However, researchers in the field of bladder failure seem to have much work to do in the future.

- Conflict of Interest: No potential conflict of interest relevant to this article was reported.

\section{REFERENCES}

1. Griffiths DJ. Editorial: bladder failure--a condition to reckon with. J Urol 2003;169:1011-2.

2. Osman NI, Chapple CR, Abrams P, Dmochowski R, Haab F, Nitti V, et al. Detrusor underactivity and the underactive bladder: a new clinical entity? A review of current terminology, definitions, epidemiology, aetiology, and diagnosis. Eur Urol 2014;65:389-98. 
IJ Kwon and Lee • Learning From Heart Failure: How Will We Lead Bladder Failure Into the Future?

3. Marquez J, Lee SR, Kim N, Han J. Rescue of heart failure by mitochondrial recovery. Int Neurourol J 2016;20:5-12.

4. Nevel-McGarvey CA, Levin RM, Haugaard N, Wu X, Hudson AP. Mitochondrial involvement in bladder function and dysfunction.
Mol Cell Biochem 1999;194:1-15.

5. Levin RM, Hudson AP. The molecular genetic basis of mitochondrial malfunction in bladder tissue following outlet obstruction. J Urol 2004;172:438-47. 